\title{
Level of education, lifestyle, and morbidity in two groups of white collar workers
}

\author{
Annette Leclerc, Françoise Pietri, Liliane Boitel, Jean-François Chastang, Philippe Carval, \\ Michel Blondet
}

\begin{abstract}
Study objective-The aim was to examine the relationship between level of education, lifestyle, and morbidity in two groups of male white collar workers, and to determine whether any differences found could be related to objective differences in working conditions.
\end{abstract}

Design-The study was a survey of a random sample of commercial travellers and a sample of men with sedentary occupations, representing two different groups of white collar workers. Survey interviews were conducted during the annual compulsory medical examination. Subjects were classified into three levels of education and differences according to level of education were studied in relation to 40 frequent health problems, lifestyle variables, body mass index, height, and working conditions.

Subjects-There were 1364 men in the commercial traveller group, mean age 39.5 years, and 525 men in the sedentary group, mean age 36.2 years. There were 22 exclusions because of unclassifiable levels of education and four refused to be interviewed.

Setting-The study took place in 11 towns in France.

Main results-When age was taken into account there were only minor differences in the prevalence of health disorders. Lifestyle variables and height were clearly related to the level of education. Observed differences could not be explained by constraints or declared difficulties in working conditions.

Conclusions-Differences in health practices related to level of education are observed even in groups that are relatively homogeneous socially. Lifestyle may be important as an intermediate determinant of health disorders among less educated people.

INSERM U88,

91 Boulevard de

1'Hôpital, 75013 Paris,

France

A Leclerc

F Pietri

J-F Chastang

P Carval

CISME, 31 rue

Médéric, 75017 Paris,

France

L Boitel

M Blondet

Correspondence to:

Dr Leclerc

Accepted for publication January 1992 frequently more strongly associated with disease than are other indicators ${ }^{5}$; for example, the level of education has been found to be the best predictor of coronary heart disease. ${ }^{8}$ The causes of observed inequalities have been discussed by many investigators. ${ }^{9-13}$ The true effect of level of education, controlling for socioeconomic status and occupation, is not usually studied because these variables are most often closely interrelated.

Few results based on large samples have been published concerning the differences caused by education in an otherwise homogeneous socioeconomic group. This paper examines the relationship between the level of education and morbidity and selected risk factors in two groups of male white collar workers; a group of 519 sedentary workers of varying social backgrounds, and a group of 1348 commercial travellers. All the employees in the latter group were classified in the same socioeconomic group and occupation but the group was not homogeneous with regard to level of education (from primary school to university diplomas). Commercial travellers form a large group of employees in France: 450000 male workers out of 14 million active men.

Survey data were collected in these two groups in order to study health problems and working conditions in commercial travellers in comparison with the group of sedentary workers. We present here an additional study, focused on differences according to the level of education in the two groups. The objectives of the study were as follows.

(1) We sought to determine whether a relationship between the level of education and health problems and lifestyle risk factors was observed in the two groups. A complementary objective was to compare the strength of the associations with of level of education between the two groups, one of which was rather heterogeneous (sedentary workers), and the other more homogeneous (commercial travellers).

(2) In addition we set out to describe the living and working conditions of various subgroups in order to determine which explanations for the observed differences were plausible. Living and working conditions in these two groups are similar to those experienced by all subjects in the healthy active population without specific occupational hazards.

The study was restricted to men because the group of available women was smaller and included part time workers. Moreover, specific problems would be encountered in interpreting results restricted to active women, due to health selection with regard to women's participation in the labour force. ${ }^{14}$ 


\section{Methods}

DESIGN OF THE STUDY

A cross sectional survey was conducted from the beginning of October 1985 to the end of September 1986 in 11 towns in France in order to study occupational risk factors and morbidity of commercial travellers in comparison with a control group of white collar workers. The study was conducted, in cooperation with research workırs, by a group of physicians responsible for the medical surveillance of the workers. In France, most employees undergo a compulsory medical examination each year, performed by physicians specialised in occupational health. Seventy four of these physicians, working in the same organisation (CISME), participated in the study. They were responsible for employees with a large range of occupations, including commercial travellers. Each person in the survey was interviewed during the annual medical examination. The interview, lasting 10 to 20 minutes, was performed by the physician.

It included questions about working conditions, lifestyle, and a list of health disorders. Past history $(\mathrm{H})$, present disorders $(\mathrm{P})$, and present treatment $(T)$ were distinguished. Some present disorders were only based on symptoms (Ps), with a diagnosis considered as probable or plausible by the physician who undertook both the interview and a clinical examination. Present disorders were classified as objective ( $\mathrm{Po}$ ) when the diagnosis was based on an objective source, such as an $x$ ray or a laboratory test. However, except for blood pressure, and a simple routine test for diabetes, objective tests were not systematically performed.

The sampling procedure was as follows: each physician participating in the survey had a quota of sedentary workers and commercial travellers to interview for each quarter of the year. The first persons in each half day of routine examination to meet the inclusion criteria were included until the quota for the quarter was reached. This sampling procedure was simple and avoided recruitment bias because the arrival order of employees relied on administrative procedures, and was not under control of the physicians.

Interviews were carried out on 2571 persons: 1705 commercial travellers (1364 males) and 866 sedentary white collar workers ( 525 males). Only four persons refused to answer the questionnaire. The sample was representative of the target population in the study towns, since the physicians who volunteered to participate in the study had a similar case load to their colleagues.

The survey was conducted in addition to the annual medical examination. Eight meetings with about 10 physicians and the researchers were conducted in 1984 and 1985 in order to prepare the protocol and the questionnaire. Four other meetings were organised during each quarter of the year of data collection, in order to verify that uniform standards were being applied by the physicians. In addition the physicians in the group had local meetings with their colleagues in the town, and they were in charge locally of the quality and comparability of the data collection.

The main results of the study have been presented in a report. ${ }^{15}$
THE TWO GROUPS OF WHITE COLLAR WORKERS Neither group included self employed workers because these subjects do not benefit from annual medical examinations.

The inclusion criteria for commercial travellers were: (1) being in an employment which involves soliciting for orders and selling goods, products, and services to retailers, industrial consumers, institutional and professional establishments, or private persons; (2) visiting clients for more than half of their time.

The International standard classification of occupations ${ }^{16}$ would classify persons in this group in categories 4.32 (Commercial travellers and manufacturers' agents), 4.31 (Technical salesmen and service advisers) and 4.41 (Insurance, real estate, and securities salesmen).

All individuals in this group have similar working conditions. They are responsible for an alloted geographical area, which involves many hours driving and working far from home, sometimes for more than a day. They must eat at restaurants, alone or with clients. Some of them have heavy samples of goods to carry, although physical constraints are not as severe as in most groups of blue collar workers.

The inclusion criteria for the control group, called here sedentary workers were: (1) they should be white collar workers; (2) their working conditions should not include many hours driving, physical constraints, or night work. The most frequent occupations in the group were engineers $(n=100)$, technicians $(n=80)$, bank or insurance clerical workers $(n=66)$, and accountants $(n=43)$.

\section{ANALYSIS}

The exact level of education was known for each subject. The subjects were classified into three groups according to the level of education: L (low), less than the French baccalaureat (the diploma at the end of secondary school); $M$ (medium), the French baccalaureat or equivalent; $\mathrm{H}$ (high) university level or diplomas higher than the baccalaureat. These limits were selected in order to have three groups of similar size. It is worth noting that the so called "low" level of education in fact represents between 9 and 13 years of school.

Twenty two men were excluded because they could not be classified. The present analysis is based on 1348 commercial travellers and 519 sedentary workers.

The frequency of 40 health disorders (shown in table I) was compared according to the level of education separately for commercial travellers and sedentary workers. The list of health disorders was restricted to those with a prevalence of more than $4 \%$ in at least one of the groups. The limit of $4 \%$ was selected as it allowed comparisons of percentages to be performed in the two groups (except for two health disorders in the group of sedentary workers). The list included being on a diet prescribed by a physician, and sick leave within the past 12 months. Table I also shows the mean age in each subgroup.

The relationship between the level of education and general risk factors was studied separately in the two groups. The variables, presented in table II, are related to lifestyle (tobacco, alcohol, sport), body mass index, and height. Height was included 
since this variable is generally associated with level of education and is sometimes considered as a risk indicator. ${ }^{917}$

The relationship between level of education and working conditions was also studied in the two groups. The list of working conditions in table IV is based on previous comparisons between commercial travellers and sedentary workers. In the first study ${ }^{15}$ these variables were considered as giving a good description of working conditions in the group of commercial travellers, in comparison with the group of sedentary workers.

Finally, a complementary analysis was performed in order to clarify the relationship between lifestyle risk factors, level of education, and working conditions.

Comparisons were made with $\chi^{2}$ tests for qualitative variables, and variance analysis for quantitative variables. For infrequent health disorders, only two levels of education were considered (low versus medium + high). Comparisons were not performed if the number of cases was not large enough for a $\chi^{2}$ test. The effect of age was controlled for using a logistic model for dichotomous variables, and two way analysis of variance for quantitative variables. For age, three groups were considered ( $<35,35-45,>45$ years). This part of the analysis was restricted for health disorders to situations in which the $\chi^{2}$ test was significant.

A logistic model was also used in the last part of the analysis in order to study associations between education and lifestyle, controlling for some working conditions and age.

\section{Results}

HEALTH DISORDERS

The numbers of subjects, the mean age in each level of education, and the frequencies of health disorders are given in table I.
Table I Frequency of health disorders according to level of education

\begin{tabular}{|c|c|c|c|c|c|c|c|c|c|c|c|}
\hline & & \multicolumn{5}{|c|}{ Commercial travellers } & \multicolumn{5}{|c|}{ Sedentary workers } \\
\hline & & \multicolumn{3}{|c|}{$\begin{array}{l}\text { Frequency by level } \\
\text { of education }\end{array}$} & \multirow[b]{2}{*}{ Test 1} & \multirow[b]{2}{*}{ Test 2} & \multicolumn{3}{|c|}{$\begin{array}{l}\text { Frequency by level } \\
\text { of education }\end{array}$} & \multirow[b]{2}{*}{ Test 1} & \multirow[b]{2}{*}{ Test 2} \\
\hline & & $\begin{array}{l}L \\
(n=592\end{array}$ & $\begin{array}{l}M \\
(n=366)\end{array}$ & $\begin{array}{c}H \\
(n=383)\end{array}$ & & & $\begin{array}{l}L \\
(n=219)\end{array}$ & $\begin{array}{l}M \\
(n=102)\end{array}$ & $\begin{array}{l}H \\
(n=196)\end{array}$ & & \\
\hline \multicolumn{2}{|l|}{ Mean age (years) } & $41 \cdot 1$ & 37.9 & $38 \cdot 8$ & $\mp$ & & $37 \cdot 4$ & $36 \cdot 6$ & $35 \cdot 0$ & $\star$ & \\
\hline $\begin{array}{l}\text { Musculoskeletal disord } \\
\text { Neck } \\
\text { Upper back } \\
\text { Low back } \\
\text { Shoulders } \\
\text { Elbow, wrist, hand } \\
\text { Knee, ankle, foot }\end{array}$ & $\begin{array}{l}\text { ders } \\
\text { H } \\
\text { PS } \\
\text { H } \\
\text { PS } \\
\text { H } \\
\text { PS } \\
\text { PO } \\
\text { T } \\
\text { H } \\
\text { PS } \\
\text { H } \\
\text { PS } \\
\text { H } \\
\text { PS } \\
\text { PO }\end{array}$ & $\begin{array}{r}12.0 \\
13.0 \\
7.8 \\
7.3 \\
30.7 \\
26.5 \\
7.8 \\
9.1 \\
6.6 \\
7.6 \\
4.6 \\
6.1 \\
12.3 \\
10.8 \\
4.4\end{array}$ & $\begin{array}{r}12 \cdot 3 \\
10 \cdot 9 \\
9 \cdot 0 \\
8 \cdot 7 \\
28 \cdot 1 \\
24 \cdot 6 \\
9 \cdot 3 \\
8 \cdot 7 \\
6 \cdot 6 \\
4 \cdot 4 \\
5 \cdot 5 \\
4 \cdot 6 \\
10 \cdot 7 \\
10 \cdot 1 \\
4 \cdot 1\end{array}$ & $\begin{array}{r}10.7 \\
10.7 \\
12.5 \\
7.6 \\
28.7 \\
25.1 \\
5.7 \\
6.5 \\
5 \cdot 2 \\
5 \cdot 2 \\
6 \cdot 3 \\
4 \cdot 7 \\
12.3 \\
9.7 \\
3.4\end{array}$ & $\begin{array}{l}\text { NS } \\
\text { NS } \\
\text { NS } \\
\text { NS } \\
\text { NS } \\
\text { NS } \\
\text { NS } \\
\text { NS } \\
\text { NS } \\
\text { NS } \\
\text { NS } \\
\text { NS } \\
\text { NS } \\
\text { NS }\end{array}$ & $\begin{array}{l}\overline{-} \\
\text { NS } \\
\bar{z} \\
\bar{z} \\
\bar{z} \\
\bar{z} \\
\bar{z} \\
\overline{-}\end{array}$ & $\begin{array}{r}5.5 \\
3.7 \\
6 \cdot 8 \\
3.7 \\
21.9 \\
16 \cdot 4 \\
7.8 \\
8 \cdot 2 \\
3.7 \\
3.2 \\
3.7 \\
1.4 \\
8.7 \\
6.8 \\
3.2\end{array}$ & $\begin{array}{r}6.9 \\
7.8 \\
8.8 \\
6.9 \\
18.6 \\
10.8 \\
6.9 \\
2.9 \\
3.9 \\
3.9 \\
4.9 \\
6.9 \\
7.8 \\
5.9 \\
1.0\end{array}$ & $\begin{array}{r}7 \cdot 7 \\
5 \cdot 6 \\
6.1 \\
3 \cdot 1 \\
18.9 \\
15.3 \\
4 \cdot 1 \\
3 \cdot 1 \\
3 \cdot 1 \\
2 \cdot 6 \\
6 \cdot 1 \\
2.6 \\
11.7 \\
8.2 \\
6 \cdot 1\end{array}$ & $\begin{array}{l}\text { NS } \\
\text { NS } \\
\text { NS } \\
\text { (NS) } \\
\text { NS } \\
\text { NS } \\
\text { NS } \\
\text { (NS) } \\
\text { (NS) } \\
\text { (NS) } \\
\text { (NS) } \\
\text { NS } \\
\text { NS } \\
\text { (NS) }\end{array}$ & $\begin{array}{l}\overline{-} \\
\overline{-} \\
\overline{-} \\
\overline{-} \\
\pm \\
\overline{-} \\
\overline{-} \\
\overline{-} \\
\overline{-}\end{array}$ \\
\hline $\begin{array}{l}\text { Digestive disorders } \\
\text { Ulcers } \\
\text { Other oesophagus, } \\
\text { stomach }\end{array}$ & H & 7.6 & 5.5 & $2 \cdot 6$ & $\dagger$ & * & $5 \cdot 0$ & 0.0 & $3 \cdot 6$ & (NS) & - \\
\hline $\begin{array}{l}\text { Bowel } \\
\text { Liver, gallbladder }\end{array}$ & $\begin{array}{l}\mathrm{H} \\
\mathrm{PS} \\
\mathrm{H} \\
\mathrm{PS} \\
\mathrm{H}\end{array}$ & $\begin{array}{c}8 \cdot 1 \\
11 \cdot 1 \\
8 \cdot 1 \\
9 \cdot 3 \\
5.9\end{array}$ & $\begin{array}{l}8 \cdot 5 \\
9 \cdot 0 \\
8 \cdot 5 \\
9 \cdot 0 \\
3 \cdot 8\end{array}$ & $\begin{array}{l}8 \cdot 1 \\
7 \cdot 3 \\
7 \cdot 3 \\
9 \cdot 7 \\
3 \cdot 7\end{array}$ & $\begin{array}{l}\text { NS } \\
\text { NS } \\
\text { NS } \\
\text { NS } \\
\text { NS }\end{array}$ & $\begin{array}{l}\overline{-} \\
\bar{z} \\
\bar{y}\end{array}$ & $\begin{array}{l}8 \cdot 7 \\
7 \cdot 8 \\
6 \cdot 4 \\
7 \cdot 3 \\
7 \cdot 3\end{array}$ & $\begin{array}{l}4.9 \\
7.8 \\
6.9 \\
5.9 \\
5.9\end{array}$ & $\begin{array}{l}2 \cdot 0 \\
4 \cdot 1 \\
5 \cdot 6 \\
7 \cdot 1 \\
5 \cdot 6\end{array}$ & $\begin{array}{l}\star \\
\text { NS } \\
\text { NS } \\
\text { NS } \\
\text { NS }\end{array}$ & $\begin{array}{l}\star \\
\bar{z} \\
- \\
-\end{array}$ \\
\hline $\begin{array}{l}\text { Metabolic disorders } \\
\text { Lipids } \\
\text { Uric acid }\end{array}$ & $\begin{array}{l}\text { H } \\
\text { PO } \\
\text { PT } \\
\text { H }\end{array}$ & $\begin{array}{l}9 \cdot 5 \\
6 \cdot 1 \\
5 \cdot 1 \\
4.9\end{array}$ & $\begin{array}{l}7 \cdot 1 \\
4 \cdot 4 \\
3 \cdot 6 \\
4 \cdot 4\end{array}$ & $\begin{array}{l}4 \cdot 7 \\
3.9 \\
3 \cdot 1 \\
4 \cdot 2\end{array}$ & $\begin{array}{l}\star \\
\text { NS } \\
\text { NS } \\
\text { NS }\end{array}$ & $\begin{array}{l}\text { NS } \\
- \\
-\end{array}$ & $\begin{array}{l}5.5 \\
3.7 \\
1.8 \\
2.3\end{array}$ & $\begin{array}{l}2.9 \\
4.9 \\
2 \cdot 0 \\
2.9\end{array}$ & $\begin{array}{l}3.1 \\
1.5 \\
2.0 \\
1.5\end{array}$ & $\begin{array}{l}\text { (NS) } \\
\text { (NS) } \\
-\end{array}$ & $\begin{array}{l}\overline{-} \\
\overline{-}\end{array}$ \\
\hline $\begin{array}{l}\text { Cardiovascular disorde } \\
\text { Hypertension } \\
\text { Varicose veins } \\
\text { Haemorroids }\end{array}$ & $\begin{array}{c}\text { lers } \\
\text { H } \\
\text { or T } \\
\text { H } \\
\text { PO } \\
\text { H } \\
\text { PS } \\
\text { PO } \\
\text { T }\end{array}$ & $\begin{aligned} 8 \cdot 3 \\
9 \cdot 3 \\
4 \cdot 2 \\
5 \cdot 1 \\
12.2 \\
6 \cdot 8 \\
3 \cdot 9 \\
4 \cdot 1\end{aligned}$ & $\begin{array}{r}6 \cdot 6 \\
8 \cdot 5 \\
3 \cdot 3 \\
4 \cdot 1 \\
14 \cdot 8 \\
10 \cdot 4 \\
4 \cdot 9 \\
4 \cdot 4\end{array}$ & $\begin{array}{r}4 \cdot 2 \\
3.9 \\
1 \cdot 8 \\
3.4 \\
11 \cdot 7 \\
8 \cdot 4 \\
3 \cdot 9 \\
4 \cdot 4\end{array}$ & $\begin{array}{l}\star \\
\dagger \\
\text { NS } \\
\text { NS } \\
\text { NS } \\
\text { NS } \\
\text { NS } \\
\text { NS }\end{array}$ & $\begin{array}{l}\text { NS } \\
\text { NS } \\
= \\
= \\
= \\
= \\
-\end{array}$ & $\begin{array}{l}6 \cdot 4 \\
8 \cdot 2 \\
7 \cdot 3 \\
8 \cdot 7 \\
5 \cdot 9 \\
5 \cdot 0 \\
1 \cdot 8 \\
1 \cdot 8\end{array}$ & $\begin{array}{l}3.9 \\
3.9 \\
2 \cdot 0 \\
1.0 \\
6.9 \\
2 \cdot 9 \\
1 \cdot 0 \\
2 \cdot 0\end{array}$ & $\begin{array}{l}1.5 \\
1.0 \\
2 \cdot 6 \\
2.0 \\
9.7 \\
5.1 \\
2 \cdot 6 \\
2.0\end{array}$ & 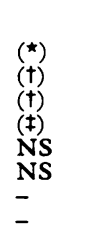 & $\begin{array}{l}\text { NS } \\
\star \\
\text { NS } \\
\dagger \\
- \\
= \\
-\end{array}$ \\
\hline $\begin{array}{l}\text { Respiratory disorders } \\
\text { Asthma }\end{array}$ & H & $3 \cdot 5$ & $2 \cdot 7$ & $6 \cdot 0$ & NS & - & $5 \cdot 5$ & $6 \cdot 9$ & $5 \cdot 6$ & NS & - \\
\hline $\begin{array}{l}\text { Head and neck disorde } \\
\text { Minor disorders } \\
\text { Upper respiratory } \\
\text { Hearing disorders }\end{array}$ & $\begin{array}{l}\text { lers } \\
\text { H } \\
\text { PO } \\
\text { H } \\
\text { PS } \\
\text { H }\end{array}$ & $\begin{array}{l}5 \cdot 4 \\
6 \cdot 8 \\
6 \cdot 1 \\
5.9 \\
4 \cdot 1\end{array}$ & $\begin{array}{l}3 \cdot 3 \\
4 \cdot 1 \\
5 \cdot 7 \\
5 \cdot 2 \\
2 \cdot 7\end{array}$ & $\begin{array}{l}4 \cdot 2 \\
5 \cdot 0 \\
6 \cdot 5 \\
5 \cdot 7 \\
4 \cdot 2\end{array}$ & $\begin{array}{l}\text { NS } \\
\text { NS } \\
\text { NS } \\
\text { NS } \\
\text { NS }\end{array}$ & $\begin{array}{l}\bar{z} \\
\bar{z} \\
\bar{y}\end{array}$ & $\begin{array}{l}4 \cdot 6 \\
5 \cdot 9 \\
4 \cdot 6 \\
2 \cdot 7 \\
2 \cdot 3\end{array}$ & $\begin{array}{l}4 \cdot 9 \\
5 \cdot 9 \\
4 \cdot 9 \\
2 \cdot 9 \\
8 \cdot 8\end{array}$ & $\begin{array}{l}5 \cdot 6 \\
7 \cdot 1 \\
3 \cdot 1 \\
3 \cdot 1 \\
3 \cdot 6\end{array}$ & $\begin{array}{l}\text { NS } \\
\text { NS } \\
\text { (NS) }\end{array}$ & $\begin{array}{l}\bar{z} \\
\bar{z} \\
\bar{y}\end{array}$ \\
\hline $\begin{array}{l}\text { Urogenital disorders } \\
\text { Lithiasis }\end{array}$ & $\mathbf{H}$ & $6 \cdot 1$ & $6 \cdot 6$ & $5 \cdot 2$ & NS & - & $3 \cdot 7$ & $4 \cdot 9$ & $5 \cdot 6$ & (NS) & - \\
\hline Diet, medical reasons & & $17 \cdot 3$ & $12 \cdot 4$ & $9 \cdot 0$ & $\ddagger$ & NS & 13.8 & 6.9 & $4 \cdot 6$ & $\dagger$ & * \\
\hline Sick leave & & $22 \cdot 4$ & 17.5 & 17.5 & NS & - & $21 \cdot 7$ & $16 \cdot 0$ & $19 \cdot 0$ & NS & - \\
\hline
\end{tabular}

$\mathrm{L}, \mathrm{M}, \mathrm{H}=$ low, medium, high education (see text); $\mathrm{H}=$ past history; $\mathrm{PS}=$ present disorders (symptoms); $\mathrm{PO}=$ present objective disorders; $T=$ treatment

Test $1=$ comparison of percentages; Test $2=$ relationship with level of education, controlling for age (based on logistic model): ${ }^{\star} \mathrm{p}<0.05 ; \mathrm{t} p<0.01 ; \neq \mathrm{p}<0.001 ;()=$ test with only two levels of education: low $v$ medium + high; $-=$ test not performed 
A significant relationship with the level of education was observed for only six health disorders among commercial travellers (of 42 tests performed). When age was taken into account only one health disorder remained significantly more frequent among the less educated (past history of ulcers). Among sedentary workers, 38 comparative tests could be performed. Seven health disorders were more frequent among less educated men. The association remained significant for five disorders when age was taken into account: past history of stomach and oesophageal disorders (except for ulcers), present hypertension, and medical diet were related to the level of education with a clear gradient; treatment for low back pain and varicose veins were more frequent in the group of less educated men.

RISK FACTORS ASSOCIATED WITH LIFESTYLE

Table II shows the means and frequencies of lifestyle risk factors and height according to the level of education.

The level of education was associated with many of these variables, especially in the group of commercial travellers. Subjects with the lowest level of education were smaller and heavier (in relation to their height). They drank more wine and aperitifs, and engaged in sports less often. No relationship with tobacco consumption was observed.

When age was controlled for, most associations persisted (tables II and III). However, engaging in sport was no longer significant for sedentary workers, and the test for body mass index was borderline in both groups $(p=0.07)$.

WORKING CONDITIONS

Table IV shows some aspects of the working and living conditions in the six subgroups. They can be classified according to the kind of effect they are expected to have:

(1) The first group (physical constraints) includes working conditions which could have a direct effect on specific health disorders. These are variables related to physical load, previously found to be related to musculoskeletal disorders met by commercial travellers. ${ }^{15}$

(2) The second group (lifestyle constraints) includes constraints on meals and absence from home which could affect prevalence and intensity of some risk factors, for example, obesity, alcohol consumption, and the lack of sport.

(3) The third group (stressful conditions) includes opinions of subjects about the many adverse aspects of their work. These could reflect workload, or level of insecurity in work, or difficulties for some subjects in coping with the constraints of work. The variables in this list are percentages of subjects who answered "yes" to the question: "In your work, which of the following conditions are hard to bear for you?"

The relationships between the level of education and working conditions were different according to the kind of constraints. The results were only slightly different when age was taken into account. Thus physical constraints were more often met among less educated workers, while on the other hand employees with the lowest level of education did not suffer more frequently from constraints affecting lifestyle. For commercial travellers, the relationship with customers often involves business meals, which give a greater opportunity for overeating and drinking alcohol. However, this type of meal was more frequent in the group with the highest level of education.

The results concerning stressful conditions were more relevant to the commercial travellers
Table II Lifestyle variables, height, and body mass index according to level of education
Table III Odds ratios (OR) associated with levels of education and lifestyle variables (controlling for age in three trolling for age in three
classes: $<35,35-45$, $>45$ years)

\begin{tabular}{|c|c|c|c|c|c|c|c|c|c|c|}
\hline & \multicolumn{5}{|c|}{ Commercial travellers } & \multicolumn{5}{|c|}{ Sedentary workers } \\
\hline & \multicolumn{3}{|c|}{$\begin{array}{l}\text { Frequency by level } \\
\text { of education }\end{array}$} & \multirow[b]{2}{*}{ Test 1} & \multirow[b]{2}{*}{ Test 2} & \multicolumn{3}{|c|}{$\begin{array}{l}\text { Frequency by level } \\
\text { of education }\end{array}$} & \multirow[b]{2}{*}{ Test 1} & \multirow[b]{2}{*}{ Test 2} \\
\hline & $\begin{array}{l}L \\
(n=592)\end{array}$ & $\begin{array}{l}M \\
(n=366)\end{array}$ & $\begin{array}{l}H \\
(n=383)\end{array}$ & & & $\begin{array}{l}L \\
(n=219)\end{array}$ & $\begin{array}{l}M \\
(n=102)\end{array}$ & $\begin{array}{l}H \\
(n=196)\end{array}$ & & \\
\hline $\begin{array}{l}\text { Height }(\mathrm{cm}) \\
\text { Body mass index }\left(\mathrm{kg} / \mathrm{m}^{2}\right)\end{array}$ & $\begin{array}{r}173 \cdot 4 \\
25 \cdot 3\end{array}$ & $\begin{array}{r}175 \cdot 0 \\
24 \cdot 7\end{array}$ & $\begin{array}{r}176 \cdot 5 \\
24 \cdot 2\end{array}$ & $\begin{array}{l}\ddagger \\
\ddagger\end{array}$ & $\begin{array}{l}\ddagger \\
\mathrm{p}=0.07\end{array}$ & $\begin{array}{r}173 \cdot 3 \\
24 \cdot 5\end{array}$ & $\begin{array}{r}174 \cdot 7 \\
23 \cdot 7\end{array}$ & $\begin{array}{r}174 \cdot 4 \\
23 \cdot 5\end{array}$ & $\begin{array}{l}t \\
t\end{array}$ & $\begin{array}{l}t \\
p=0.07\end{array}$ \\
\hline $\begin{array}{l}\text { Smokers }(\%) \\
\text { Smokers > 20/day (\%) } \\
\text { Ex-smokers }(\%)\end{array}$ & $\begin{array}{l}51.7 \\
10.9 \\
12.5\end{array}$ & $\begin{array}{l}52 \cdot 2 \\
11.9 \\
10 \cdot 7\end{array}$ & $\begin{array}{r}55 \cdot 2 \\
9 \cdot 1 \\
7 \cdot 8\end{array}$ & $\begin{array}{l}\text { NS } \\
\text { NS } \\
\text { NS }\end{array}$ & $\begin{array}{l}\text { NS } \\
\text { NS } \\
\text { NS }\end{array}$ & $\begin{array}{r}43 \cdot 1 \\
9 \cdot 6 \\
11.0\end{array}$ & $\begin{array}{r}43 \cdot 1 \\
4 \cdot 9 \\
13 \cdot 7\end{array}$ & $\begin{array}{r}43 \cdot 4 \\
5 \cdot 7 \\
8 \cdot 2\end{array}$ & $\begin{array}{l}\text { NS } \\
\text { NS } \\
\text { NS }\end{array}$ & $\begin{array}{l}\text { NS } \\
\text { NS } \\
\text { NS }\end{array}$ \\
\hline $\begin{array}{l}\text { Wine, } \geqslant 4 \text { glasses/day (\%) } \\
\text { Aperitifs, }\end{array}$ & $34 \cdot 9$ & $26 \cdot 6$ & $17 \cdot 1$ & $\ddagger$ & $\ddagger$ & $27 \cdot 1$ & $19 \cdot 6$ & $11 \cdot 2$ & $\ddagger$ & $t$ \\
\hline $\begin{array}{l}\geqslant 1 \text { glass/day }(\%) \\
\geqslant 2\end{array}$ & $\begin{array}{r}21 \cdot 7 \\
7.1\end{array}$ & $\begin{array}{r}17 \cdot 4 \\
5 \cdot 2\end{array}$ & $\begin{array}{r}10 \cdot 8 \\
2 \cdot 1\end{array}$ & $\begin{array}{l}\ddagger \\
\dagger\end{array}$ & $\begin{array}{l}\ddagger \\
\dagger\end{array}$ & $\begin{array}{r}11 \cdot 6 \\
3 \cdot 2\end{array}$ & $\begin{array}{r}15 \cdot 7 \\
2 \cdot 9\end{array}$ & $\begin{array}{l}8 \cdot 2 \\
0.5\end{array}$ & $\begin{array}{l}\text { NS } \\
\text { NS }\end{array}$ & $\begin{array}{l}\text { NS } \\
\text { NS }\end{array}$ \\
\hline Engage in sport $(\%)$ & $36 \cdot 4$ & $41 \cdot 6$ & $53 \cdot 3$ & $\ddagger$ & $\ddagger$ & $36 \cdot 1$ & $49 \cdot 0$ & $47 \cdot 7$ & $\star$ & NS \\
\hline
\end{tabular}

$\mathrm{L}, \mathrm{M}, \mathrm{H}=$ low, medium, high education (see text)
Test $\mathrm{I}=$ comparison of percentages or analysis of variance; Test $2=$ relationship with level of education, controlling for age. Based on two way analysis of variance (means) or logistic model (percentages): ${ }^{\star} \mathrm{p}<0.05 ; \dagger \mathrm{p}<0.01 ; \neq \mathrm{p}<0.001$

\begin{tabular}{|c|c|c|c|c|c|c|c|c|}
\hline & \multicolumn{4}{|c|}{ Commercial travellers } & \multicolumn{4}{|c|}{ Sedentary workers } \\
\hline & \multicolumn{3}{|c|}{$\begin{array}{l}\text { OR by level } \\
\text { of education }\end{array}$} & \multirow[b]{2}{*}{ Test } & \multicolumn{3}{|c|}{$\begin{array}{l}\text { OR by level } \\
\text { of education }\end{array}$} & \multirow[b]{2}{*}{ Test } \\
\hline & $L$ & $M$ & $H(1)$ & & $L$ & $M$ & $H(1)$ & \\
\hline $\begin{array}{l}\text { Smoker } \\
\text { Smokers >20 g/day } \\
\text { Ex-smokers }\end{array}$ & $\begin{array}{l}1 \cdot 03 \\
1 \cdot 30 \\
1 \cdot 24\end{array}$ & $\begin{array}{l}0.95 \\
1.38 \\
1.25\end{array}$ & $\begin{array}{l}1.00 \\
1.00 \\
1.00\end{array}$ & $\begin{array}{l}\text { NS } \\
\text { NS } \\
\text { NS }\end{array}$ & $\begin{array}{l}1.05 \\
1.69 \\
1.27\end{array}$ & $\begin{array}{l}1.01 \\
0.81 \\
1.68\end{array}$ & $\begin{array}{l}1.00 \\
1.00 \\
1.00\end{array}$ & $\begin{array}{l}\text { NS } \\
\text { NS } \\
\text { NS }\end{array}$ \\
\hline $\begin{aligned} \text { Wine, } \geqslant 4 \text { glasses/day } \\
\text { Aperitifs, } \\
\text { श1 glass/day } \\
\geqslant 2\end{aligned}$ & $\begin{array}{l}2 \cdot 11 \\
2 \cdot 41 \\
3 \cdot 13\end{array}$ & $\begin{array}{l}1.63 \\
1.76 \\
2.44\end{array}$ & $\begin{array}{l}1.00 \\
1.00 \\
1.00\end{array}$ & $\begin{array}{l}\ddagger \\
\pm \\
\dagger\end{array}$ & $\begin{array}{l}2 \cdot 60 \\
1 \cdot 45 \\
7 \cdot 16\end{array}$ & $\begin{array}{l}1 \cdot 76 \\
2 \cdot 02 \\
5 \cdot 65\end{array}$ & $\begin{array}{l}1 \cdot 00 \\
1.00 \\
1 \cdot 00\end{array}$ & $\begin{array}{l}\dagger \\
\text { NS } \\
\text { NS }\end{array}$ \\
\hline Engage in sport & 0.57 & 0.66 & 1.00 & $\ddagger$ & 0.68 & 1.09 & $1 \cdot 00$ & NS \\
\hline
\end{tabular}

$\mathrm{L}, \mathrm{M}, \mathrm{H}=$ low, medium, high education (see text); (1) = reference category

${ }^{\star} \mathrm{p}<0.05 ; \mathrm{t} \mathrm{p}<0.01 ; \neq \mathrm{p}<0.001$ 
than to the sedentary workers because the questions were specially adapted to the former group. Some aspects of working conditions were clearly more stressful for subjects with a low level of education, for example "relationships with customers" and "objectives to reach". This refers to difficulties in striking a bargain, and the effects of the present economic crisis, which in turn relates to job insecurity. In the group of sedentary workers, significant associations were found for subjects with a high level of education with regard to relationship with "customers", and "personal work load". "Personal work load" was significantly more often declared as hard to bear among the most educated subjects in both groups of workers.

LEVEL OF EDUCATION, LIFESTYLE, AND STRESSFUL CONDITIONS

The results described above raised the question as to whether the main determinant of negative aspects of lifestyle was stressful conditions at work rather than the level of education per se. This hypothesis was tested in the group of commercial travellers, and restricted to variables for which this form of association might exist, as indicated by the relationships observed in the first part of the analysis.

Four logistic models were used, with the same explanatory variables: level of education, age, and presence or absence of three stressful conditions: "relationships with customers", "effects of economic crisis", and "objective to reach". The outcome variables were: wine, four glasses per day; aperitifs, $\geqslant 1$ glass per day; aperitifs, $\geqslant 2$ glasses per day; practice of a sport.

The results concerning the level of education are given in table $\mathrm{V}$, with a presentation similar to that in table III. The comparison between table $\mathrm{V}$ and table III indicates that controlling for stressful conditions did not modify the relationship with the level of education, which remains the main determinant of these lifestyle variables.

The models did not suggest negative effects of stressful conditions on lifestyle. The only signifi-
Table $V$ Odds ratios (OR) associated with level of education controlling for age and three stressful conditions (commercial travellers only): relations with customers, economic crisis, and objectives to reach

\begin{tabular}{lllll}
\hline & \multicolumn{3}{l}{ OR by level of education } & \\
\cline { 2 - 4 } & $L$ & $M$ & $H(1)$ & Test \\
\hline Wine, $\geqslant 4$ glasses/day & 2.13 & 1.63 & 1.00 & $\ddagger$ \\
Aperitifs $\geqslant 1$ glass/day & 2.36 & 1.76 & 1.00 & $\ddagger$ \\
Aperitifs $\geqslant 2$ glass/day & 3.25 & 2.49 & 1.00 & $\dagger$ \\
Practice of a sport & 0.58 & 0.67 & 1.00 & $\ddagger$
\end{tabular}

$\mathrm{L}, \mathrm{M}, \mathrm{H}=$ low, medium, high level of education (see text); (1) = reference category $\mathrm{tp}<0.01 ; \neq \mathrm{p}<0.00$

cant association was in the opposite direction: absence of complaints about "relationships with customers" was associated with a higher frequency of daily alcohol coonsumption.

\section{Discussion}

It can be considered that no major bias affected the results, since the main objective of the study was not to compare levels of education, which minimises the interviewer bias. Only four persons refused to be interviewed; the exact level of education was known, and probably well declared to an occupational physician; among lifestyle variables, alcohol consumption was probably underreported (as in many studies). However, comparisons concerning this variable are consistent with known results concerning social differences in consumption, and expected results such as the higher consumption among commercial travellers as a whole, compared to other white collar workers.

Interpretation of the results must take into account the fact that the study was restricted to active men with at least a basic level of instruction and income, and equal access to health facilities.

The fact that the groups did not include blue collar workers partly accounts for the results which showed only limited differences in health disorders and no differences in tobacco according to the level of education. With regard to morbidity among commercial travellers, there may be differential health selection bias according to level of
Table IV Working conditions according to level of education

\begin{tabular}{|c|c|c|c|c|c|c|c|c|c|c|}
\hline & \multicolumn{5}{|c|}{ Commercial travellers } & \multicolumn{5}{|c|}{ Sedentary workers } \\
\hline & \multicolumn{3}{|c|}{$\begin{array}{l}\text { Frequency by level } \\
\text { of education }\end{array}$} & \multirow[b]{2}{*}{ Test 1} & \multirow[b]{2}{*}{ Test 2} & \multicolumn{3}{|c|}{$\begin{array}{l}\text { Frequency by level } \\
\text { of education }\end{array}$} & \multirow[b]{2}{*}{ Test 1} & \multirow[b]{2}{*}{ Test 2} \\
\hline & $\bar{L}$ & $M$ & $H$ & & & $L$ & $M$ & $H$ & & \\
\hline $\begin{array}{l}\text { Physical constraints (\% y } \\
\text { Carrying loads } \\
\text { Standing } \\
\text { Stairs to climb }\end{array}$ & $\begin{array}{l}\text { es) } \\
34.9 \\
52.0 \\
28 \cdot 1\end{array}$ & $\begin{array}{l}27 \cdot 7 \\
45 \cdot 8 \\
32 \cdot 3\end{array}$ & $\begin{array}{l}29 \cdot 4 \\
34 \cdot 9 \\
32 \cdot 3\end{array}$ & $\begin{array}{l}\star \\
\pm \\
\text { NS }\end{array}$ & $\begin{array}{l}\text { NS } \\
\ddagger \\
\text { NS }\end{array}$ & $\begin{array}{l}25 \cdot 2 \\
43 \cdot 1 \\
30 \cdot 3\end{array}$ & $\begin{array}{l}11.9 \\
22.8 \\
17.8\end{array}$ & $\begin{array}{r}8 \cdot 2 \\
26 \cdot 0 \\
27 \cdot 0\end{array}$ & $\begin{array}{l} \pm \\
\ddagger \\
\text { NS }\end{array}$ & $\begin{array}{l}\ddagger \\
\ddagger \\
\text { NSS }\end{array}$ \\
\hline $\begin{array}{l}\text { Lifestyle and work } \\
\text { Irregular hours of } \\
\text { meals }(\%) \\
\text { Unbalanced meals (\%) } \\
\text { Business meals/quarter }\end{array}$ & $\begin{array}{l}47.0 \\
43.0\end{array}$ & $\begin{array}{l}44 \cdot 5 \\
42 \cdot 9\end{array}$ & $\begin{array}{l}41 \cdot 8 \\
41 \cdot 0\end{array}$ & $\begin{array}{l}\text { NS } \\
\text { NS }\end{array}$ & $\begin{array}{l}\text { NS } \\
\text { NS }\end{array}$ & $\begin{array}{l}10 \cdot 0 \\
18 \cdot 3\end{array}$ & $\begin{array}{l}14.9 \\
14 \cdot 7\end{array}$ & $\begin{array}{r}8.2 \\
16.8\end{array}$ & $\begin{array}{l}\text { NS } \\
\text { NS }\end{array}$ & $\begin{array}{l}\text { NS } \\
\text { NS }\end{array}$ \\
\hline $\begin{array}{l}\text { (mean) } \\
\text { Hotel night/month }\end{array}$ & 4.5 & 6.7 & 8.8 & $\ddagger$ & $\ddagger$ & 0.9 & 3.8 & $3 \cdot 4$ & - & - \\
\hline (mean) & $4 \cdot 8$ & 4.4 & 4.9 & NS & NS & 0.2 & 0.8 & $0 \cdot 6$ & - & - \\
\hline $\begin{array}{l}\text { Stressful conditions (\% ye } \\
\text { Relationships with } \\
\text { customers } \\
\text { Hierarchical constraints } \\
\text { Loneliness } \\
\text { Being far from family } \\
\text { Economic crisis } \\
\text { Personal work load } \\
\text { Objectives to reach }\end{array}$ & $\begin{array}{l}\text { es) } \\
11 \cdot 7 \\
15 \cdot 1 \\
22 \cdot 2 \\
24 \cdot 6 \\
63 \cdot 9 \\
27 \cdot 0 \\
40 \cdot 6\end{array}$ & $\begin{array}{l}10.1 \\
15.8 \\
20.0 \\
21.0 \\
54.0 \\
30.9 \\
37.7\end{array}$ & $\begin{array}{r}5.8 \\
13.2 \\
25.3 \\
25.8 \\
44.5 \\
32.9 \\
30.9\end{array}$ & $\begin{array}{l}\dagger \\
\text { NS } \\
\text { NS } \\
\text { NS } \\
\pm \\
\text { NS } \\
t\end{array}$ & $\begin{array}{l}\star \\
\text { NS } \\
\text { NS } \\
\text { NS } \\
\pm \\
\star \\
\text { NS }\end{array}$ & $\begin{array}{r}4 \cdot 1 \\
14 \cdot 7 \\
3 \cdot 7 \\
2 \cdot 8 \\
28 \cdot 1 \\
3 \cdot 7 \\
12 \cdot 4\end{array}$ & $\begin{array}{r}6 \cdot 9 \\
12 \cdot 9 \\
5 \cdot 0 \\
6 \cdot 9 \\
30.7 \\
12 \cdot 9 \\
18 \cdot 8\end{array}$ & $\begin{array}{r}12 \cdot 2 \\
9 \cdot 7 \\
3 \cdot 6 \\
4 \cdot 1 \\
27 \cdot 2 \\
12 \cdot 8 \\
17 \cdot 3\end{array}$ & $\begin{array}{l}\dagger \\
\text { NS } \\
\text { NS } \\
\text { NS } \\
\text { NS } \\
\text { t } \\
\text { NS }\end{array}$ & $\begin{array}{l}\dagger \\
\text { NS } \\
\text { NS } \\
\text { NS } \\
\text { NS } \\
\ddagger \\
\text { NS }\end{array}$ \\
\hline
\end{tabular}


education. For tobacco consumption, an expected (although not significant) excess of heavy smokers was observed among less educated sedentary workers. Among commercial travellers, the absence of a relation has no simple explanation.

Some of the observed differences in health disorders could be due to objective conditions. For example, the higher frequency of varicose veins and treatment for low back pain among less educated men in the group of sedentary workers could be related to the fact that physical constraints (loading and frequent standing) are most frequent in this subgroup. However, a large part of observed differences, especially those related to an unhealthy way of life (alcohol consumption and lack of exercise), cannot be explained by objective conditions. For example, commercial travellers in the low level of education drink more alcohol, although they experience less constraints concerning business meals.

These results are in accordance with the importance of lifestyle as an intermediate determinant of health disorders among less educated persons, as stressed by many authors. In most developed countries, less eduated people tend to smoke more, have an incorrect diet, ${ }^{18}$ and are more often obese. $^{719}$ In France, in addition, they are more often heavy consumers of alcohol, ${ }^{20}{ }^{21}$ as in Italy, ${ }^{7}$ but unlike many other developed countries.

A relationship between the level of education and height, related to childhood conditions, is observed in many countries. ${ }^{5922}$ Height is also associated with social mobility. ${ }^{9}$ Here, this association is observed in both groups. For commercial travellers, the interpretation is complex. Firstly commercial travellers with the lowest level of education can be considerd as upwardly mobile (at least from the end of their formal education to their present occupation). Secondly, the group of commercial travellers, as a whole, has a higher mean height than sedentary workers, due to self selection of tall men for commercial travelling occupations.

The level of education remains the most important determinant of lifestyle risk factors. In the last part of the analysis we tried to test the hypothesis that unhealthy practices may be more a consequence of stressful conditions than an effect of level of education per se. The hypothesis was not verified but this could be due to inadequate variables in our data, such as complaints of subjects rather than objective working conditions. On the other hand, the analysis exhibited a counter example: among commercial travellers, an unhealthy practice such as daily consumption of an aperitif is associated with an absence of complaints about relationships with customers.
In the French context, it is understandable that drinking with customers helps to maintain good relationships with them.

This example, which certainly cannot be generalised, stresses the complexity of the relationships between the level of education, working conditions, and lifestyle, especially for lifestyle aspects closely linked to social practices.

1 Kitagawa EM, Hauser PM. Differential mortality in the United States. Cambridge, Mass: Harvard University Press, 1973.

2 Valkonen T. Adult mortality and level of education: comparison of six countries. In: Fox J, ed. Health inequalities in European countries. Aldershot: Gower inequalities in European countries.

3 Liberatos P, Link BG, Kelsey JL. The measurement of social class in epidemiology. Epidemiol Rev 1988; 10 $87-121$.

4 Abramson JH, Gofin R, Habib J, Pridan H, Gofin J. Indicators of social class: a comparative appraisal of measures for use in epidemiological studies. Soc Sci Med 1982; 16: 1739-46.

5 Doornbos G, Krommout D. Educational level and mortality in a 32-years follow up study of 18-years-old men in The Netherlands. Int f Epidemiol 1990; 19: 374-9.

6 Pincus T, Callahan LF, Burkhauser RV. Most chronic diseases are reported more frequently by individuals with fewer than 12 years of formal education in the age 18-64 United States population. $尹$ Chron Dis $1987 ; 40$ : 865-74.

7 La Vecchia C, Negri E, Pagano R, Decarli A. Education, prevalence of disease, and frequency of health care prevalence of disease, and frequency of health care
utilisation. $f$ Epidemiol Community Health 1987; 41: 161-5.

8 Jacobsen BK, Thelle SS. Risk factors for coronary heart Jacobsen BK, Thelle SS. Risk factors for coronary heart
disease and level of education: the Tromso Heart Study. disease and level of education: the

9 Fogelman K, Fox AJ, Power C. Class and tenure mobility: do they explain social inequalities in health among young adults in Britian? In: Fox J, ed. Health inequalities in European countries. Aldershot: Gower Publishing Company, 1989: 333-52.

10 Hart N. Inequalities in health: the individual versus the environment. F $R$ Stat Soc A 1986; 149: 228-46.

11 Illsley R. Occupational class, selection and the production of inequalities. F Soc Affairs 1985; 2: 151-65.

12 Fox AJ, Goldblatt PO, Jones DR. Social class differentials: artefact, selection or life circumstances? $\mathcal{f}$ Epidemiol Community Health 1985; 39: 1-8.

13 Marmot MG, Shipley MJ, Rose G. Inequalities in death, specific explanations of a general pattern? Lancet 1984; specific

14 Arber S. Gender and class inequalities in health understanding the differentials. In: Fox J, ed. Health understanding the differentials. In: Fox J, ed. Health
inequalities in European countries. Aldershot: Gower inequalities in European countries.

15 Boitel L, Arnaud F, Bardot F, et al. Les forces de vente. Relations sante-travail. Etude tranversale, document No 7/1990. Paris: CISME, 1990.

16 International Labour Office. International standard classification of occupations. Geneva: ILO, 1968.

17 Walker M, Shaper AG, Wannamathee G. Height and socia class in middle-aged British men. $\mathcal{F}$ Epidemiol Community Health 1988; 42: 299-303.

18 Braddon FEM, Wadsworth MEJ, Davies JMC, Cripps HA. Social and regional differences in food and alcohol consumption and their measurement in a national birth cohort. $\mathcal{F}$ Epidemiol Community Health 1988; 42: 341-9.

19 Braddon FEM, Rodgers B, Wadsworth MEJ, David JMC. Onset of obesity in a 36 year birth cohort study. BMF 1986; 293: $299-303$.

20 Lelaidier S. Quelques résultats sur les consommateurs de boissons alcooliques et de tabac en France en 1980. Informations Rapides 1983; 20: 1-12.

21 Guignon N. Alcool et tabac, des plaisirs qui empoisonnent ... In: Données sociales. Paris: INSEE 1990.

22 Douglas JWB, Simpson HR. Height in relation to puberty family size and social class: a longitudinal study. Milbank Memorial Fund $Q$ 1964; 42: 20-35. 